\title{
Beam loss monitoring with unfolding techniques
}

\author{
M. Caresana ${ }^{1, a} \mathbb{D}$, M. Ferrarini ${ }^{2}$, M. Frosini ${ }^{1}$, M. Reginatto ${ }^{3}$ \\ ${ }^{1}$ Department of Energy, Politecnico di Milano, via Lambruschini, 4, 20156 Milan, Italy \\ 2 Fondazione CNAO, Strada Privata Campeggi, 27100 Pavia, Italy \\ 3 Physikalisch-Technische Bundesanstalt, Bundesallee 100, 38116 Brunswick, Germany
}

Received: 6 December 2020 / Accepted: 7 February 2021

(C) The Author(s) 2021

\begin{abstract}
Since the first particle accelerator's construction in 1931, an exponential spread of these machines occurred worldwide, in different kinds of applications. Nowadays, these are mainly used for industrial $(60 \%)$ and medical (35\%) purposes and for scientific research (5\%). High energy secondary mixed fields produced by the particle beams interaction with matter imply a complex environmental dosimetry and special radiation protection regulations able to guarantee workers and population safety. In the medical field, this aspect is particularly emphasized in hadrontherapy centres, where high energy charged particles such as protons and carbon ions modify environmental doses, with a significant increase in the neutron contribution. This work proposes a technique to identify points of losses of the primary particle beam around an acceleration ring and has been developed within the radiation protection section at the National Centre for Oncological Hadrontherapy situated in Pavia. In the first part, the radiation field produced by protons and carbon ions interactions with structural materials at different energies was investigated. The main instrument of analysis is the Monte Carlo code for particle transport FLUKA, supported by experimental measurements in the treatment room carried out with the rem counter LUPIN, designed for pulsed neutron fields dosimetry. This first step allowed an analysis of both the angular and energetic instrumental response and a comparison of experimental results with simulations. The second part proposes a description of the technique for beam loss positions reconstruction around the acceleration ring at CNAO based on the application of unfolding codes.
\end{abstract}

\section{Introduction}

Hadrontherapy is a specific radiotherapy technique developed in 1939 which uses hadrons, such as neutrons and protons, to treat oncological diseases. Treatments with proton beam were proposed by R. Wilson in 1946; after several years of technological studies the first protontherapy facility was built and currently there are facilities worldwide. A well-shaped conformation dose in the target region can be obtained with protons and carbon ions. In particular, carbon ions can maximize the cellular damage because their LET and RBE are higher than for protons [1].

The main effect of the interaction of high charged particles with matter is the production of electromagnetic and intra-nuclear cascades, with the consequent emission of high energy

\footnotetext{
a e-mail: marco.caresana@polimi.it (corresponding author)
} 
particles concentrated within the original direction of the projectiles. A second process occurs at the end of the intra-nuclear cascade. The nucleus remains in an excited state and de-excitate with the emission of evaporation neutrons with energy around $1 \mathrm{MeV}$. Neutrons and photons represent the major contribution to this process when the energy of the primary beam is below few hundreds of $\mathrm{MeV}$. Energetic neutrons, coupled with photons, propagate for long distances inside the materials, until they reach fractions of $\mathrm{eV}$ and can be absorbed [2]. The hadron acceleration up to the energy required for hadrontherapy (250 MeV for protons and $400 \mathrm{MeV} / \mathrm{amu}$ for carbon ions) is done with synchrotrons or cyclotrons. For carbon ions synchrotrons acceleration is the only option, and cyclotrons can only be used for protons. During the acceleration process, a wide range of phenomena can cause a partial or total beam loss along the synchrotron pipeline: interactions of the beam with structural materials and orbit instabilities are some examples. Specific losses are typically localized in the injection and extraction sections, involving a limited number of bunches of primary particles, leading to a beam loss whose duration is in the range of ns [3,4]. The secondary neutron and photon radiation, produced by the beam loss, maintains the same time structure leading to a burst of radiation with a duration in the range of ns. The measurement of beam losses is a central problem in particle accelerators and is faced in very different ways and with beam loss monitors (BLMs) based on different detectors. At J-PARC main ring [5] more than 200 BLMs composed by ion chamber and proportional counters are placed along the about $1.6 \mathrm{~km}$ long ring. The space resolution is very high, about 1 BLM every $7 \mathrm{~m}$, permitting a precise identification of the loss point. At SwissFEL [6] the beam loss monitoring is based on secondary neutron measurement and the instrument of choice was the LUPIN (see below) because of its extremely wide dynamic in terms of neutron dose per single radiation burst. At ESS [7] the BLM system measures fast neutrons and the monitor of choice is the Micromegas detector, specifically designed to be sensitive to fast neutrons and intrinsically insensitive to photons and the relative response of the detectors deployed along the LINAC provides information on the loss location. The only suggestion to face the problem of identifying beam losses with unfolding techniques is reported in [8], where the BLMs around LHC are described, providing also a matrix containing the BLM signal for different loss points. Such representation is useful for possible unfolding of the observed BLM signals and respective translation into loss points. The aim of this work is to introduce an innovative procedure able to identify the positions around the synchrotron where the beam loss occurs. The secondary radiation burst generated by a single beam loss is measured at a few fixed positions around the accelerator and the beam loss position is identified by unfolding these data with GRAVEL $[9,10]$, an unfolding program based on an iterative algorithm that is commonly used for Bonner sphere spectrometry data. The advantage of this technique is to greatly reduce the number of BLMs deployed along the acceleration ring.

The main challenge of measuring the mixed radiation bursts is in the short duration and in the separation of the neutron and photon components. To address this issue, the decision was to use the commercial rem counter LUPIN (see Sect. 2.3) distributed by ElseNuclear S.r.L.-Italy. This detector demonstrated a superior capability to measure neutrons in pulsed and mixed fields, when compared to other rem counters. To obtain the response function to be used in the unfolding code, the LUPIN response to beam losses has been simulated with the Monte Carlo radiation transport code FLUKA. 


\section{Materials and methods}

\subsection{The accelerator at CNAO}

There are only six hadrontherapy facilities where tumoral diseases can be treated both with protons and carbon ions. One is the CNAO (Centro Nazionale di Adroterapia Oncologica), located in Pavia, Italy. Its construction lasted from 2005 to 2010 and the first successful treatment with protons took place in 2011 and with carbon ions 1 year later. The machine used to accelerate particles at CNAO is a synchrotron of 25 m radius. (Fig. 1)

Two ECR sources produce $H^{+}$and $C^{6+}$ ions which are pre-accelerated to $7 \mathrm{MeV} / \mathrm{u}$ within a LINAC; these particles are then injected in the main accelerator ring which can accelerate protons from 60 up to $250 \mathrm{MeV}$ and carbon ions from 120 up to $400 \mathrm{MeV} / \mathrm{u}$ [11]. The particle beam is extracted in spills and can be sent to one of the three treatment rooms: all these rooms have a horizontal line with an additional vertical line for the central one. The approximate number of particles per spill is $6 \times 10^{9}$ for protons and $3 \times 10^{8}$ for carbon ions.

\subsection{The Monte Carlo code FLUKA}

The computational analysis was done with the Monte Carlo code FLUKA for particle transport and interaction, which can manage about 60 different particles such as photons, electrons, hadrons, fast and thermal neutrons in a range of energy from fractions of eV up to $\mathrm{TeV}$ [12]. The neutrons transport from thermal energy up to $20 \mathrm{MeV}$ is based on a multi-group algorithm, which includes more than 260 different ranges of energy, 31 only for thermal neutrons. A dedicated library contains cross sections of about 250 different materials, including different types of reactions.

\subsection{The rem counter LUPIN}

The instrumentation used for the measurement of the neutron bursts is the neutron detector LUPIN (Long Interval Ultra-wide dynamic Pile-up free Neutron rem counter), based on a proportional counter, filled with BF3, with a logarithmic amplification front-end electronics able to measure a single neutron interaction.

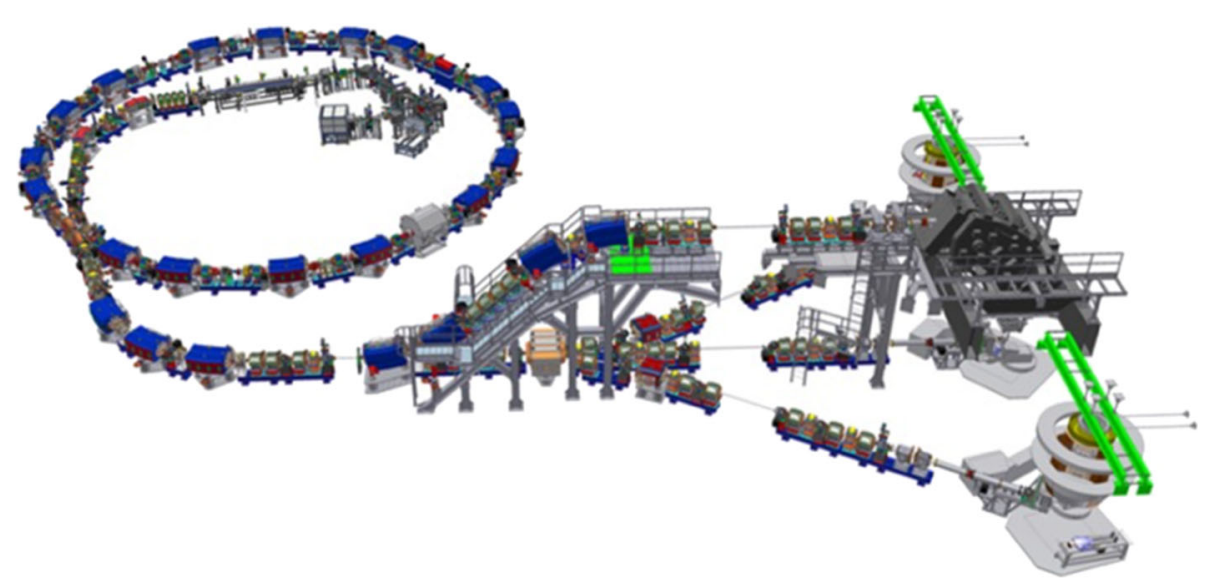

Fig. 1 Structure of the accelerator installed at CNAO 

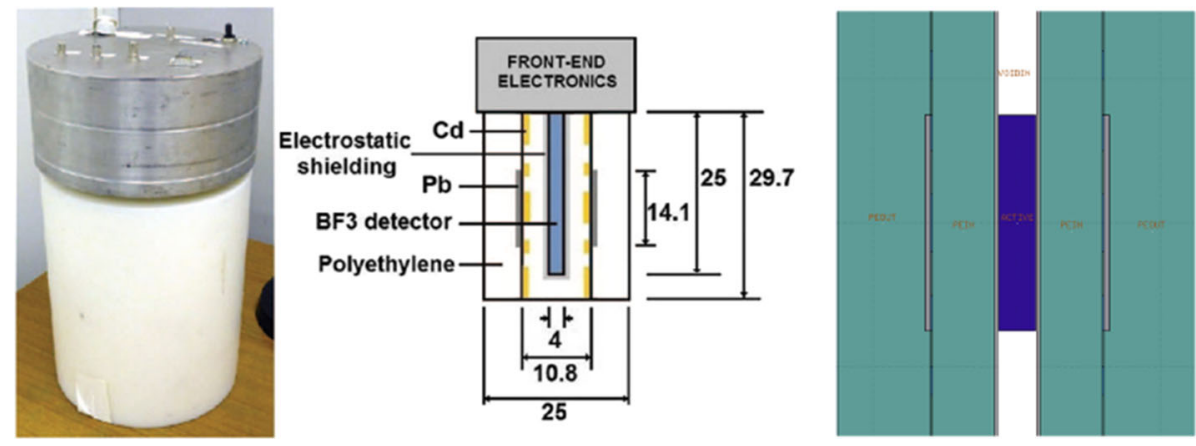

Fig. 2 Structure of neutron dosimeter LUPIN and simulated geometry in FLUKA

This device has been specifically developed to measure the neutron contribution in high energy pulsed mixed fields produced, for example, around an accelerator. The linearity of the LUPIN, with respect to the neutron burst intensity, has been measured up to a dose of about $500 \mathrm{nSv} /$ burst that is more than one order of magnitude higher than conventional rem counters [13]. The moderator region (Fig. 2) is constituted by a $25 \mathrm{~cm}$ diameter cylinder made of polyethylene, with special inserts made of cadmium and lead. The detector is installed inside this region, with an electrostatic shield of aluminium $1.5 \mathrm{~mm}$ thick $[14,15]$. The output signal is acquired by a FPGA and then processed with a dedicated software. Two different types of data acquisition are available: in streaming mode a mean collected charge (MCC), produced by neutron interactions, is calculated and an evaluation of $\mathrm{H}^{*}(10)$ is performed, while the derivative mode is used to optimize $\gamma / \mathrm{n}$ discrimination, when high intensity photon fields are present [16].

\subsection{The unfolding technique}

The unfolding problem that we consider is formulated in terms of the linear equation

$$
M_{n}=R_{n, m} * P_{m},
$$

where $M_{n}$ is the vector of measurements and $n=1, \ldots, N$ labels the detectors, $P_{m}$ is the vector of beam losses and $m=1, \ldots, M$ labels the beam loss positions, and $R_{n, m}$ plays the role of a response matrix that relates the vector of beam losses to the vector of measurements. Both $M_{n}$ and $R_{n, m}$ are known; thus, the goal of the unfolding is to determine a physically meaningful $P_{m}$ that solves this equation. The analysis was carried out using the few-channel unfolding program GRAVEL. This standard program, which is well known from applications in Bonner sphere spectrometry, is a modification of the earlier iterative unfolding program SAND-II and it may be considered a particular type of nonlinear least-squares adjustment algorithm that enforces positivity [10]. The implementation of GRAVEL that we used is the one of the program GRV-FC33 which is available as part of the UMG package [9]. 


\section{Results and discussion}

\subsection{Lupin results}

In order to evaluate the instrumentation response at different angles and energies, associated with mixed fields produced by particle beam interactions with the structural materials, a series of FLUKA simulations have been performed. Particle beams at different energies (60, $150,200,250 \mathrm{MeV}$ for protons and 120, 200, 280, $400 \mathrm{MeV} / \mathrm{u}$ for carbon ions) are simulated impinging on a copper target with eight detectors positioned at $0^{\circ}, 15^{\circ}, 30^{\circ}, 45^{\circ}, 60^{\circ}, 90^{\circ}$, $120^{\circ}$ and $150^{\circ}$ with respect to the primary beam direction. A detailed analysis in terms of different contributions with respect to the complete radiation field has been performed: for protons only the neutron contribution has been investigated, while for carbon ions also protons, heavy and light ions have been considered. As reported in Table 1, the major contribution to the secondary radiation field is concentrated around the beam particle direction: ions and protons are predominant in this region, while for angles greater than $15^{\circ}$ their contribution is negligible, and the neutron component prevails. A measurement campaign in the treatment room has been conducted to compare the simulations with the experimental data. The LUPIN, initially at $15^{\circ}$, has been moved to $45^{\circ}$ and $90^{\circ}$ afterwards. As for the computational part, four different energies and particles have been used: an example of results, compared with ones obtained from FLUKA, is reported in Fig. 3. As expected, the highest count rate is obtained at small angle and for high energy beam particles. Concerning the instrument responses, LUPIN measurements have shown good agreement with simulated values at all the angles and energies tested. The systematic underestimation of the measured data can be due to the simplification of the simulation geometry. In fact the complex geometrical structure of the magnets was assumed as a copper cylinder, neglecting material between the interaction and the measurement points that can attenuate the neutron beam. Considering the simplification, an agreement within $30 \%$ is considered more than satisfactory.

\subsection{Results of unfolding techniques to beam losses analyses}

The response function that is needed to carry out the unfolding has been evaluated by simulating eight LUPIN dosimeters around the accelerator ring each $45^{\circ}$ and simulating a beam loss

Table 1 Results of FLUKA simulations for the analysis of high energy particles mixed field components

\begin{tabular}{lllll}
\hline Angle $\left(^{\circ}\right)$ & Total & Neutron & Protons & Ions $(Z>1)$ \\
\hline 0 & $5.15 \mathrm{E}-04$ & $1.92 \mathrm{E}-04$ & $4.04 \mathrm{E}-05$ & $2.74 \mathrm{E}-04$ \\
15 & $1.38 \mathrm{E}-04$ & $1.05 \mathrm{E}-04$ & $1.12 \mathrm{E}-05$ & $1.06 \mathrm{E}-05$ \\
30 & $7.90 \mathrm{E}-05$ & $6.85 \mathrm{E}-05$ & $1.60 \mathrm{E}-05$ & - \\
45 & $6.40 \mathrm{E}-05$ & $5.80 \mathrm{E}-05$ & - & - \\
60 & $5.25 \mathrm{E}-05$ & $5.25 \mathrm{E}-05$ & - & - \\
90 & $4.04 \mathrm{E}-05$ & $4.32 \mathrm{E}-05$ & - & - \\
120 & $3.94 \mathrm{E}-05$ & $3.76 \mathrm{E}-05$ & - & - \\
150 & $3.48 \mathrm{E}-05$ & $3.46 \mathrm{E}-05$ & - & -
\end{tabular}

Fields have been investigated for protons and carbon ions impinging on a copper target. Here, the secondary particle fluence normalized per primary particle for carbon ions at $400 \mathrm{MeV} / \mathrm{u}$, for different angles with respect to the direction of primary beam 


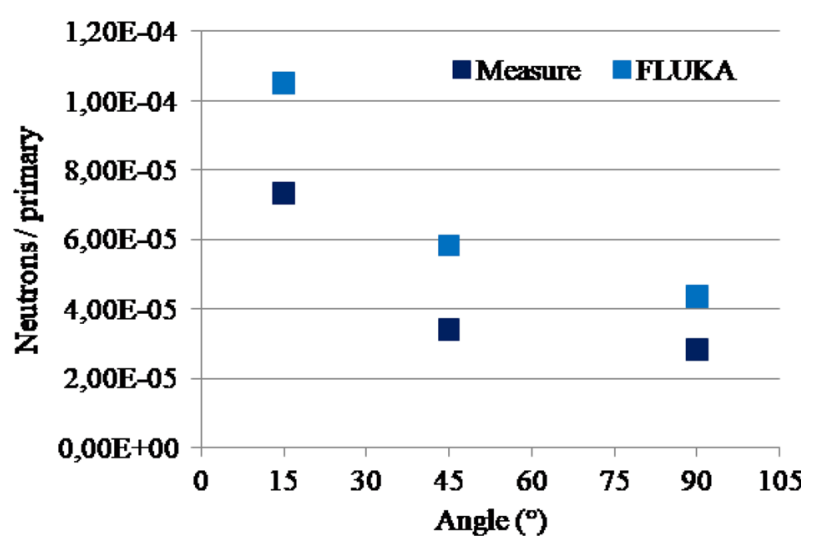

Fig. 3 Comparison between simulation and measurement results with carbon ions for LUPIN at different angles and the same energy $400 \mathrm{MeV} / \mathrm{u}$ (values normalized per primary particle)

each $5^{\circ}$ with a monoenergetic beam impinging on a copper target. The schematic structure of the simulation is shown in Fig. 4: both protons and carbon ions at the maximum energy are considered. Expected measured data from the detectors have been simulated with the same geometry, for localized beam losses and specific intensity. For the purpose of simulating the data, we have ignored fluctuations in the number of counts due to statistics and other sources of uncertainty. Such effects may be neglected here because a beam loss typically leads to a very large change in the magnitude of the signal of the detectors at locations which are close enough to be affected by the beam loss: the counts go from background to a value that can be orders of magnitude higher. Under these circumstances, fluctuations due to statistics and other effects are not sufficient to affect the main results of the unfolding.

The unfolding algorithm was then applied to the simulated data: a series of tests were performed with gradually increasing complexity. Initially, only the accelerator ring (a circle) was considered, with one or multiple beam losses, then also the extraction line was introduced and finally the unfolding was applied in the case of nominal beam losses. Concerning the initial estimate, a series of different shapes were analysed, also in this case with increasing complexity: an initial estimate similar to the correct solution, a flat initial estimate with all values equal to one, and an initial estimate with wrong loss positions. The unfolding algorithm has shown a good response for all these trials; specially in the easiest cases the algorithm was able to reconstruct at least the correct position/s of expected loss/es, while the lost particles intensity has been sometimes underestimated. As mentioned before, the starting point has been only the accelerator ring: in this case the response matrix is $8 \times 72(8$ detectors and 72 beam losses). An example of the application of the unfolding algorithm is shown in Fig. 5, in which a double beam loss with an initial estimate similar to the solution was tested. Even if in the initial estimate the intensity of lost particles has been reduced, the algorithm is able to reconstruct the correct one. After that, also the extraction line was included in simulations: in order to evaluate the response of unfolding to a no longer symmetrical geometry, a series of tests was performed also in this case. In this case the response matrix is $8 \times 80$ ( 8 additional losses for the extraction line). In Fig. 6 an example of an application of the unfolding algorithm for a double beam loss, one situated on the ring and the other on the extraction line, with a flat initial estimate, is shown. From the results two things can be noticed. First, the unfolding algorithm is able to reconstruct the correct losses positions even in a non-symmetrical geometry as it identifies two main peaks, even if with underestimated 


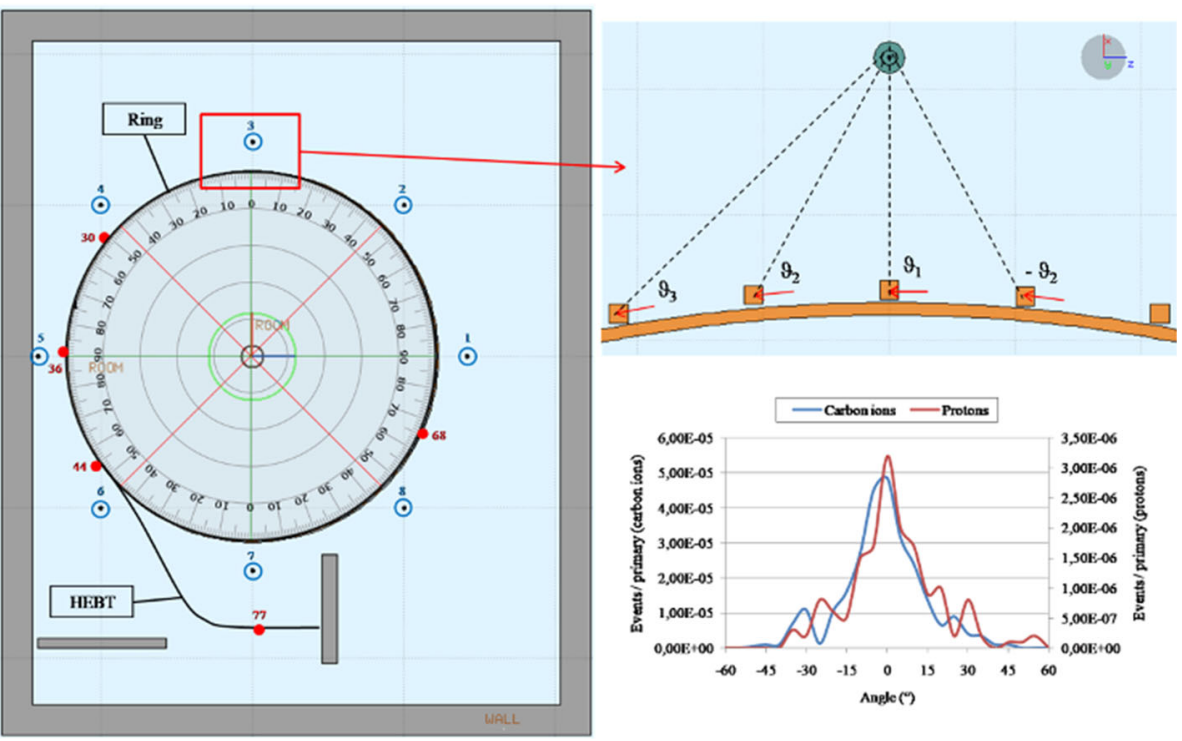

Fig. 4 Schematic structure of simulated geometry with FLUKA for response functions evaluation: blue circles are detectors, and red dots are nominal loss positions with the adopted enumeration. The single response function, depending on the beam direction, is also shown
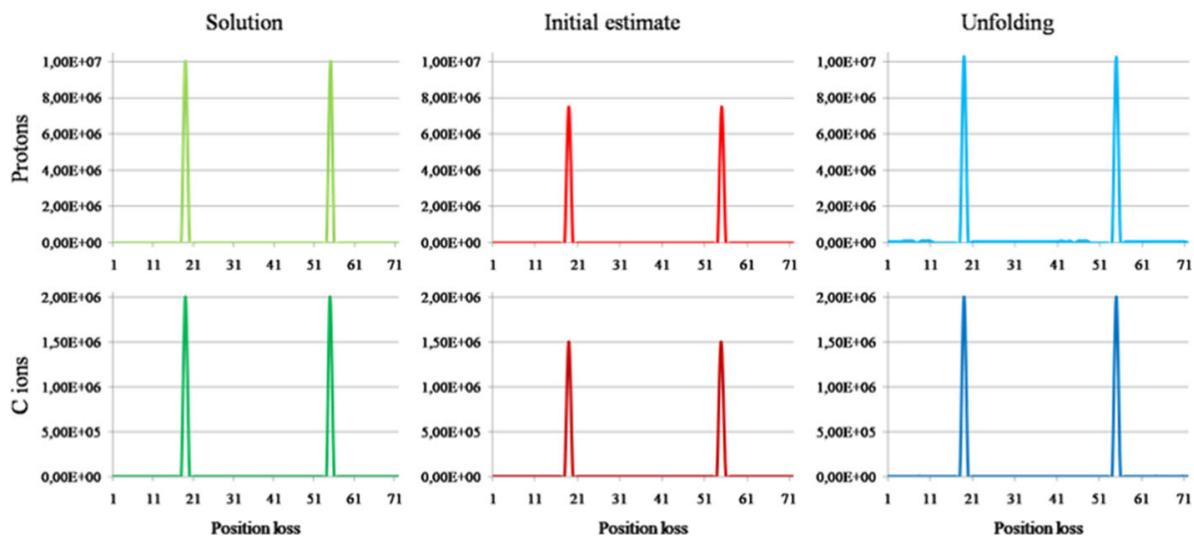

Fig. 5 Unfolding results in case of two beam losses of the same intensity, localized on the ring. The graphs labelled "solution" represent the positions where the beam losses have been simulated. The graphs labelled "initial estimate" represent the guess spectrum, that is, the starting point of the unfolding, and the graphs labelled "unfolding" represent the solution of the unfolding algorithm. Values in $Y$ axis are expressed in number of primary particles lost. In the $X$ axis is the loss position

values of lost particles intensity. A second observation can be done for the very low intensity structures indicated in Fig. 6 with a red arrow: these positions correspond to the range of simulated beam losses around 50-60 that in turn are placed along the accelerator ring near the extraction line (Fig. 4). Thus, the unfolding algorithm is capable of identifying the main positions in which losses are simulated but is not so accurate at distinguishing if lost particles are produced only along the extraction line. This aspect could be further investigated in order 

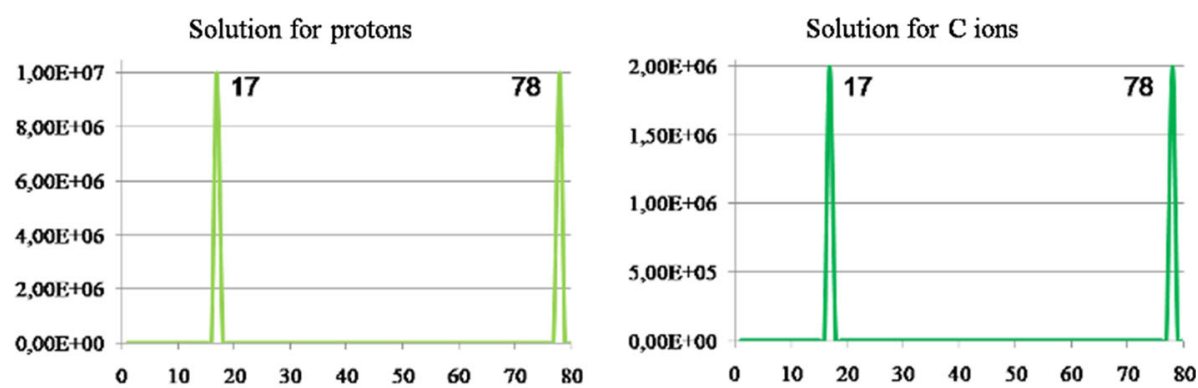

Unfolding for protons
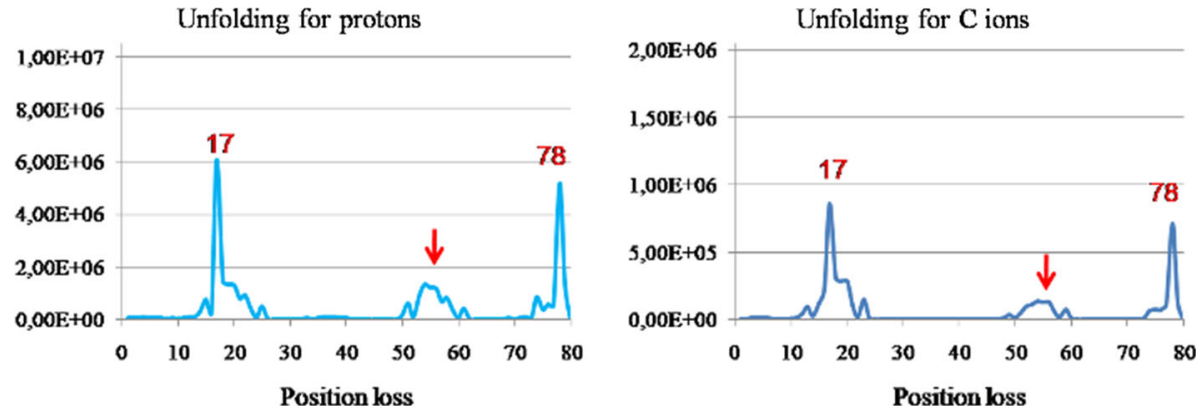

Fig. 6 Unfolding results in case of two beam losses of the same intensity, one on the ring (position 17) and one on the extraction line (position 78). The initial estimate is a flat distribution, i.e. losses uniformly distributed on all the beam loss points. The graphs labelled "solution for protons/C ions" represent the positions where beam losses are simulated. Graphs labelled "unfolding for protons/C ions" represent the output of the unfolding. Values in $Y$ axis are expressed in number of primary particles lost

to achieve a more sensitive response matrix specially in this region. As shown in Fig. 7, the unfolding procedure applied in case of all nominal beam losses around the accelerator, for a flat initial estimate, reconstructs almost the correct positions where particles are assumed to be lost. Discrepancies are present for losses too close to each other (e.g. positions 30, 36, 44) and in terms of intensity; indeed, a remarkable reduction in the number of lost particles can be observed. The unfolding solution spreads the isolated losses over a wide range of positions, with a redistribution of lost particles: despite this spectrum modification the number of lost particles integrated in a restricted range of positions is very similar to that obtained with the solution spectrum. This final consideration allows, in principle, to calculate the barycentre of the range where particles are assumed lost, and localize the most intense loss position.

\section{Conclusion and perspective}

Within this project it has been possible to investigate the secondary radiation fields generated from the interactions of particle beam with structural materials as well as their complexity in terms of energy and type of particles. In most cases the major contribution is represented by neutrons, for which a neutron dosimeter has been selected, in order to validate the simulation results. In particular, the LUPIN rem counter has shown an excellent behaviour in the presence of pulsed mixed fields. Finally, a new approach concerning the reconstruction of beam loss positions has been presented. The unfolding algorithm turned out to be a valid instrument to estimate the positions in which particles are lost: with this preliminary computational 

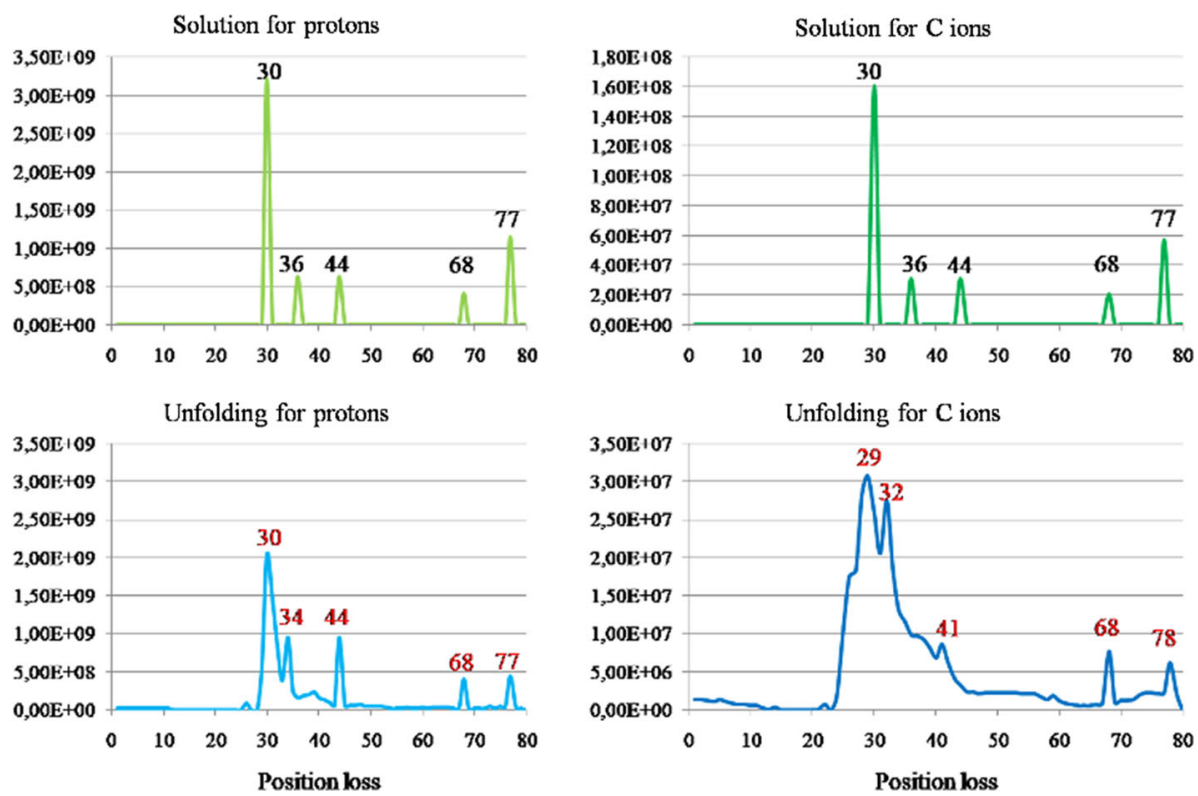

Fig. 7 Unfolding results in case of five beam losses, four of which localized in the ring and one (77) in the extraction line. The graphs labelled "solution for protons/C ions" represent the positions where beam losses are simulated. Graphs labelled "unfolding for protons/C ions" represent the output of the unfolding. Values in $Y$ axis are expressed in number of primary particles lost. In the $X$ axis is the loss position

analysis the capability of this approach to localize at least the correct positions has been shown. For a more detailed study of radiological impact related to beam losses the simulated geometry might be further modified, as well as the loss positions and lost particles fractions. Concerning the unfolding procedure, a purely computational analysis should be validated with experimental data: at first the procedure might be repeated with an updated and more realistic geometry or evaluating a new set of response functions for detectors. For the experimental part LUPIN rem counter is the most appropriate option; a set of these devices remotely controlled or, in case of a priori well knowledge of loss positions, a single one connected to a movable system, represent a possible solution.

Funding Open access funding provided by Politecnico di Milano within the CRUI-CARE Agreement.

Open Access This article is licensed under a Creative Commons Attribution 4.0 International License, which permits use, sharing, adaptation, distribution and reproduction in any medium or format, as long as you give appropriate credit to the original author(s) and the source, provide a link to the Creative Commons licence, and indicate if changes were made. The images or other third party material in this article are included in the article's Creative Commons licence, unless indicated otherwise in a credit line to the material. If material is not included in the article's Creative Commons licence and your intended use is not permitted by statutory regulation or exceeds the permitted use, you will need to obtain permission directly from the copyright holder. To view a copy of this licence, visit http://creativecommons.org/licenses/by/4.0/.

\section{References}

1. M.G. Pullia. Synchrotrons for hadrontherapy, in Reviews of Accelerator Science and Technology: Volume 2: Medical Applications of Accelerators (2009), pp. 157-178 
2. M. Pelliccioni, Fondamenti Fisici Della Radioprotezione (Pitagora editrice Bologna, Bologna, 1993)

3. R. Schmidt. Proceedings, 2014 Joint International Accelerator School: Beam Loss and Accelerator Protection (Newport Beach, CA, USA, November 5-14, 2014, Aug. 2016)

4. M. Pelliccioni, Radiation protection at Hadron therapy facilities. Radiat. Prot. Dosim. 146(4), 407-413 (2011)

5. K. Satou, T. Toyama, N. Kamikubota, S. Yoshida, J. Matsushita, T. Wakita, M. Sugiyama, T. Morino, New data acquisition system for beam loss monitor used in J-PARC main ring. Nucl. Instrum. Methods Phys. Res. Sect. A: Accel. Spectrom. Detect. Assoc. Equip. 887, 174-183 (2018)

6. C. Strabel, A. Fuchs, R. Galev, E. Hohmann, R. Lüscher, E. Musto, S. Mayer. The future SwissFEL facility-challenges from a radiation protection point of view, in EPJ Web of Conferences (vol. 153, EDP Sciences, 2017), p. 07026

7. T. Papaevangelou, H. Alves, S. Aune, J. Beltramelli, Q. Bertrand, T. Bey, B. Bolzon, N. Chauvin, M. Combet, D. Desforge, et al. ESS nBLM: Beam loss monitors based on fast neutron detection, in Proceedings of the 61st ICFA Advanced Beam Dynamics Workshop on High-Intensity and High-Brightness Hadron Beams (Daejeon, Korea, 2018), pp. 17-22

8. M. Brugger, M. Magistris, M. Santana-Leitner, F. Cerutti, V. Vlachoudis, A. Ferrari, Fluka Simulations for the Optimization of the Beam Loss Monitors (Technical Report, 2006)

9. M. Reginatto. The "few-channel" unfolding programs in the UMG package: MXD-FC33, GRV-FC33 and IQU-FC33 (UMG package, version 3.3-release date: March 1, 2004)

10. M. Matzke, Unfolding procedures. Radiat. Prot. Dosim. 107(1-3), 155-174 (2003)

11. S. Rossi, The status of CNAO. Eur. Phys. J. Plus 126(8), 78 (2011)

12. A. Fassó A. Ferrari, P.R. Sala, J. Ranft. FLUKA: A Multi-particle Transport Code (2005)

13. S. Accelerators, M. Caresana, A. Denker, A. Esposito, M. Ferrarini, N. Golnik, E. Hohmann, A. Leuschner, M. Luszik-Bhadra, G. Manessi, S. Mayer, K. Ott, J. Röhrich, M. Silari, F. Trompier, M. Volnhals, M. Wielunski, Intercomparison of radiation protection instrumentation in a pulsed neutron field. Nucl. Instrum. Methods Phys. Res. Sect. A: Detect. Assoc. Equip. 737, 203-213 (2014)

14. M. Ferrarini, V. Varoli, A. Favalli, M. Caresana, B. Pedersen, A wide dynamic range BF3 neutron monitor with front-end electronics based on a logarithmic amplifier. Nucl. Instrum. Methods Phys. Res. Sect. A: Accel. Spectrom. Detect. Assoc. Equip. 613(2), 272-276 (2010)

15. M. Caresana, M. Ferrarini, G.P. Manessi, M. Silari, V. Varoli, Lupin, a new instrument for pulsed neutron fields. Nucl. Instrum. Methods Phys. Res. Sect. A Accel. Spectrom. Detect. Assoc. Equip. 712, 15-26 (2013)

16. M. Caresana, C. Cassell, M. Ferrarini, E. Hohmann, G.P. Manessi, S. Mayer, M. Silari, V. Varoli, A new version of the lupin detector: improvements and latest experimental verification. Rev. Sci. Instrum. 85(6), 065102 (2014) 